\title{
TRANSFORMACIÓN DE LA EDUCACIÓN FRENTE A LA PANDEMIA Y LA ANALÍTICA DE DATOS
}

\section{TRANSFORMATION OF EDUCATION AGAINST PANDEMIC AND DATA ANALYTICS}

\section{Leonardo Emiro Contreras Bravo ${ }^{1}$}

\section{Héctor Javier Fuentes López²}

\section{Karolina González Guerrero 3}

Universidad Distrital Francisco José de Caldas

Universidad Militar Nueva Granada

\section{RESUMEN}

Se presenta un panorama de los cambios que está sufriendo el sector educativo de manera acelerada por la adopción de tecnologías Docente de planta. Facultad de ingeniería. Universidad Distrital Francisco José de Caldas (Colombia). lecontrerasb@ udistrital.edu.co. ORCID: https://orcid.org/0000-0003-46258835

2 Economista, Estudiante de doctorado en Economía. Docente de planta. Facultad de ingeniería. Universidad Distrital Francisco José de Caldas (Colombia). hifuentesl@udistrital.edu.co ORCID: https://orcid.org/00000001-6899-4564

3 Licenciada, Doctora en educación. Docente de planta. Facultad de estudios a distancia. Universidad Militar Nueva Granada. Karolina.gonzalez@unimilitar.edu.co kgonzalezg@gmail.com ORCID: https://orcid.org/0000-0002$\underline{\text { 9762-579X }}$

y plataformas para apoyar un proceso que tradicionalmente era presencial. Esta puede ser una oportunidad para aprovechar la información disponible de los procesos educativos y mediante el uso de técnicas como el machine learning, obtener conocimiento que permita la toma de decisiones oportunas alrededor de las problemáticas que aquejan a las Instituciones de educación. Debido a la emergencia de salud ocasionada por el COVID-19 las comunidades académicas se han visto afectadas en su salud mental evidenciando factores de ansiedad y frustración ante la nueva realidad. 
PALABRAS CLAVE: Analítica, Covid-19, Educación, Modelo, virtual

\section{SUMMARY}

It presents an overview of the changes that the education sector is undergoing in an accelerated way by adopting technologies and platforms to support a traditionally in-person process. It can be an opportunity to access the information available on educational processes and, through the use of techniques such as machine learning, obtain the knowledge that allows for timely decision-making regarding the problems that affect educational institutions. Due to the health emergency caused by COVID-19, academic communities have seen problems in their mental health, evidencing factors of anxiety, and frustration with the new reality.

KEYWORDS: Analytical, Covid-19, Education, Model, virtual

\section{INTRODUCCIÓN}

La pandemia del COVID-19 llego para cambiar el mundo. Con miles de personas en aislamiento obligatorio, el estado de los espacios digitales está cambiando de un servicio a una imperante necesidad, ya que se convierten no solo en la principal forma de acceder a la información y los servicios, sino también en uno de los únicos medios que permiten continuar con las actividades económicas, educativas, de ocio e interacciones sociales.

El reciente brote ha obligado a tomar medidas de precaución para disminuir el número de contagios, entre estas, la suspensión de clase presenciales. Miles de millones de estudiantes y jóvenes de todo el mundo se han visto afectados por los cierres temporales de colegios y universidades ordenados por los gobiernos. De esta manera el sector educativo se ha visto obligado a un cambio de paradigma en la forma de enseñar, de aprender, de socializar entre docentes, entre estudiantes y frente a lo cual la educación tradicional requiere nuevas estrategias. Surge entonces la necesidad de una respuesta para mitigar la interrupción inmediata causada por el COVID-19 y dar continuidad a las clases, así como para establecer nuevos enfoques para desarrollar sistemas de educación más abiertos y flexibles.

La cifras según la UNESCO muestran que alrededor de 1.540.000.000 de estudiantes se han visto afectados por los cierres de su institución educativa a nivel mundial, esto es el equivalente al $87.9 \%$ del total de estudiantes matriculados (UNESCO, 2020). En nuestro país, de acuerdo a las cifras del Ministerio de Educación Nacional, la cifra es de alrededor de 1.300 .000 estudiantes afectados. Por consiguiente las universidades se enfrentan al reto de explorar nuevas oportunidades con el uso de herramientas tecnologías, proyectos y análisis de información para generar un cambio de la forma de enseñar, aprender, evaluar y tomar acciones acertadas (González, 2019). Por ello se hace urgente comprender estos desafíos y preocupaciones y actuar en pro de soluciones que pueden ayudar a los estudiantes universitarios en esta crisis (Lizárraga Juárez, A., López Ramírez, E., \& Martínez Iñiguez, J. E. 2020).

Al respecto, Sanabria (2011) menciona que la integración de las TIC y los cambios hacia la innovación en la educación suponen un proceso lento, en el que la formación y actitud del docente ante estas tecnologías juegan un papel importante debido a que se requiere orientar su rol hacia su caracterización como agentes reflexivos y críticos.

La suspensión de clases en centros educativos ha obligado al uso y mediación de las Tecnologías de la información y las comunicaciones, en este escenario, los docentes son fundamentales. Para Gil Olivera, N. A. (2019) la figura del docente es vital para liderar y acompañar el proceso de cambio de identidad que las universidades se 
encuentran afrontando, con el fin de que puedan transformarse en organizaciones flexibles, posibilitando el acceso al conocimiento y la formación, adaptándose a las necesidades

Antes de la coyuntura actual, la web se venía consolidando como un espacio con gran potencial de desarrollo al facilitar la gestión de tareas como el análisis de datos, el rastreo de la información, la publicación de resultados, la gestión de proyectos, colaboración, entre otros. En el campo educativo cada vez se generan más datos gracias al uso de diversas herramientas tecnológicas como los Sistemas de Gestión de Aprendizaje (LMS) y las plataformas que permiten videoconferencias entre otros recursos, usados en diferentes universidades colombianas con el fin de integrar la enseñanza tradicional y la virtual en tiempos difíciles (Schroeder, 2009).

\section{TECNOLÓGICAS EN EDUCACIÓN}

Se han planteado diversas opciones en el marco de la sociedad de la Información que abren la posibilidad de buscar un sentido pedagógico al uso de los beneficios de la red, las ventajas de la comunicación y el análisis de la información en pro de soluciones sociales y especialmente dentro del campo educativo (Contreras, Rodriguez, \& Tarazona, 2017), más aún en tiempos de pandemia.

Varias iniciativas lanzadas en los últimos años han sido creadas como elementos fundamentales que podrían proporcionar los medios para transformar las formas en que se brinda educación y apoyo al aprendizaje, por lo que gran parte de esta actividad ha sido habilitada e inspirada por el crecimiento y la evolución de Internet. La lista de aplicaciones educativas, plataformas y recursos, que por lo general son gratuitas, y proporcionan interacción ayudando durante los períodos de cierre de colegios y universidades pueden ser clasificadas como se muestra en la Figura 1.

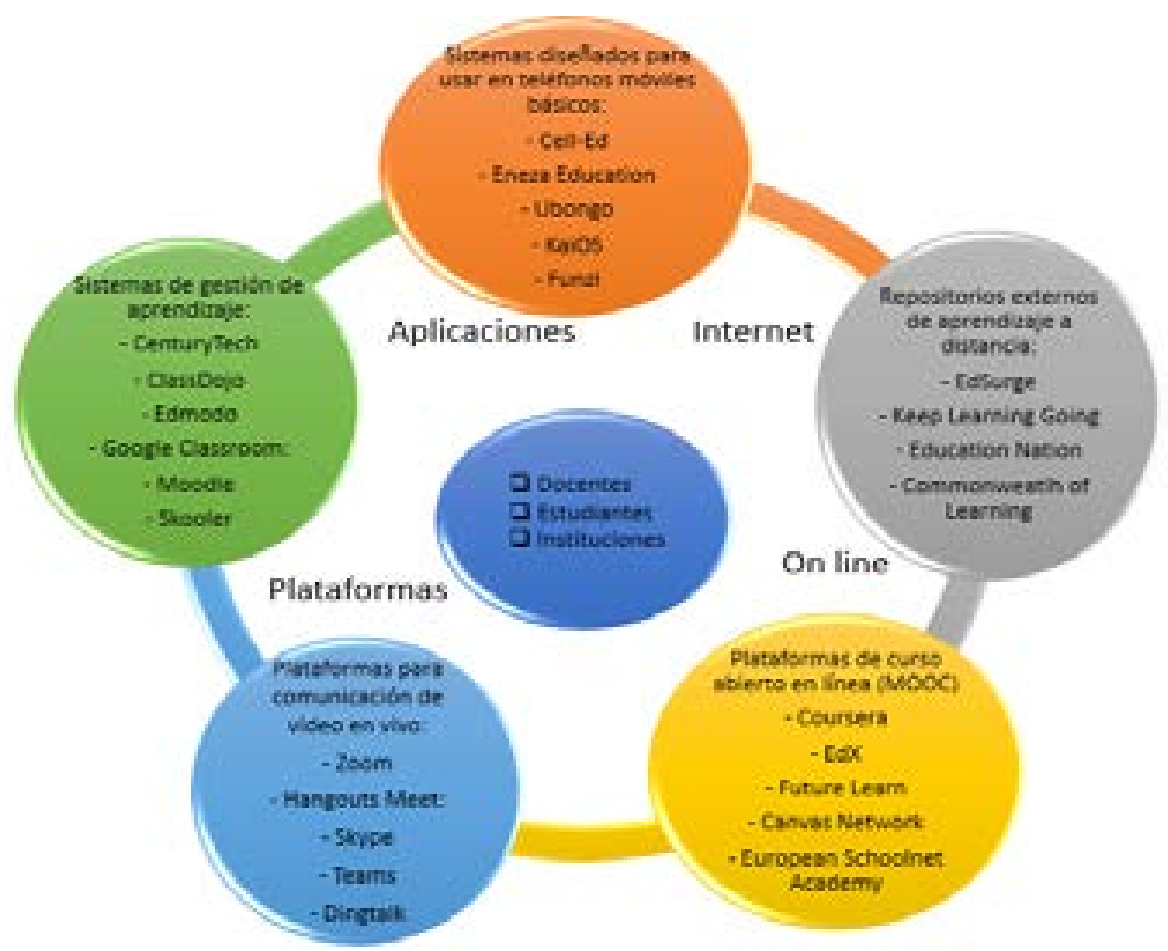

Figura 1. Aplicaciones educativas, plataformas y recursos web para educación. 
La enseñanza a través de un método tradicional como se hace hoy, está caracterizada por un trabajo en el que el docente imparte una serie de conceptos, el estudiante trata de asimilarlos y luego son evaluados (estudiante es un actor pasivo). Por tanto, parece ser el momento de apropiar nuevos conocimientos y destrezas con apoyo de tecnología donde el estudiante puede interactuar con los profesores (tutores) por medio de Internet. El ritmo acelerado del cambio en la ciencia y la ingeniería debido a los avances de la tecnología y de los retos que se plantean, hacen que se requiera altos niveles de ingenio y adaptabilidad para complementar un fuerte conocimiento del dominio y la excelencia técnica en el aprendizaje (Litzinger, Lattuca, Hadgraft, \& Newstetter, 2019).

El desafío que hoy enfrenta la educación, abre la posibilidad de buscar un sentido pedagógico al uso de los beneficios de la red respondiendo a las necesidades de una sociedad dinámica e innovadora con el fin de potencializar el proceso de enseñanza - aprendizaje en el que el estudiante pasa a ser un sujeto activo y el docente debe cambiar su rol. Ahora se ve enfrentado a un complejo escenario de paradigmas, métodos e instrumentos de evaluación así como ante una realidad generadora de datos, que se transforman y que le plantean nuevos retos de análisis de los mismos, que van más allá del simple hecho de obtener estadísticas de los procesos, sino que van causando transformaciones a nivel social, económico, político y por supuesto en el ámbito educativo (Shahiri, Husain, \& Rashid, 2015).
La tendencia hoy en las universidades es a trabajar con tecnologías que faciliten y mejoren el desarrollo de las actividades académicas, creándose de esta forma un aprendizaje electrónico añadido al tradicional (Arboleda Aparicio, J. C. 2020). El proceso de aprendizaje, ahora mediado por TIC, está generando datos que merecen ser recopilados, transformados y procesados con las herramientas adecuadas de aprendizaje automático (Machine Learning) para predecir, prevenir y/o actuar para mejorar los indicadores académicos de los estudiantes.

El aprendizaje mejorado por tecnologías (Technology enhanced learning) describe la aplicación de la tecnología a la enseñanza y el aprendizaje por medio de la generación de conocimiento a partir de una base de datos educativos. En este ámbito se han desarrollado tres enfoques de análisis de datos en el campo educativo (Figura 2): la minería de datos educativos (EDM - Educational Data Mining), la analítica académica (AA - Academic Analytics), y la analítica del aprendizaje (LA - Learning Analytics) (Ayesha, Shaeela; Mustafa, Tasleem; Sattar, Ahsan Raza; Khan, 2010). 


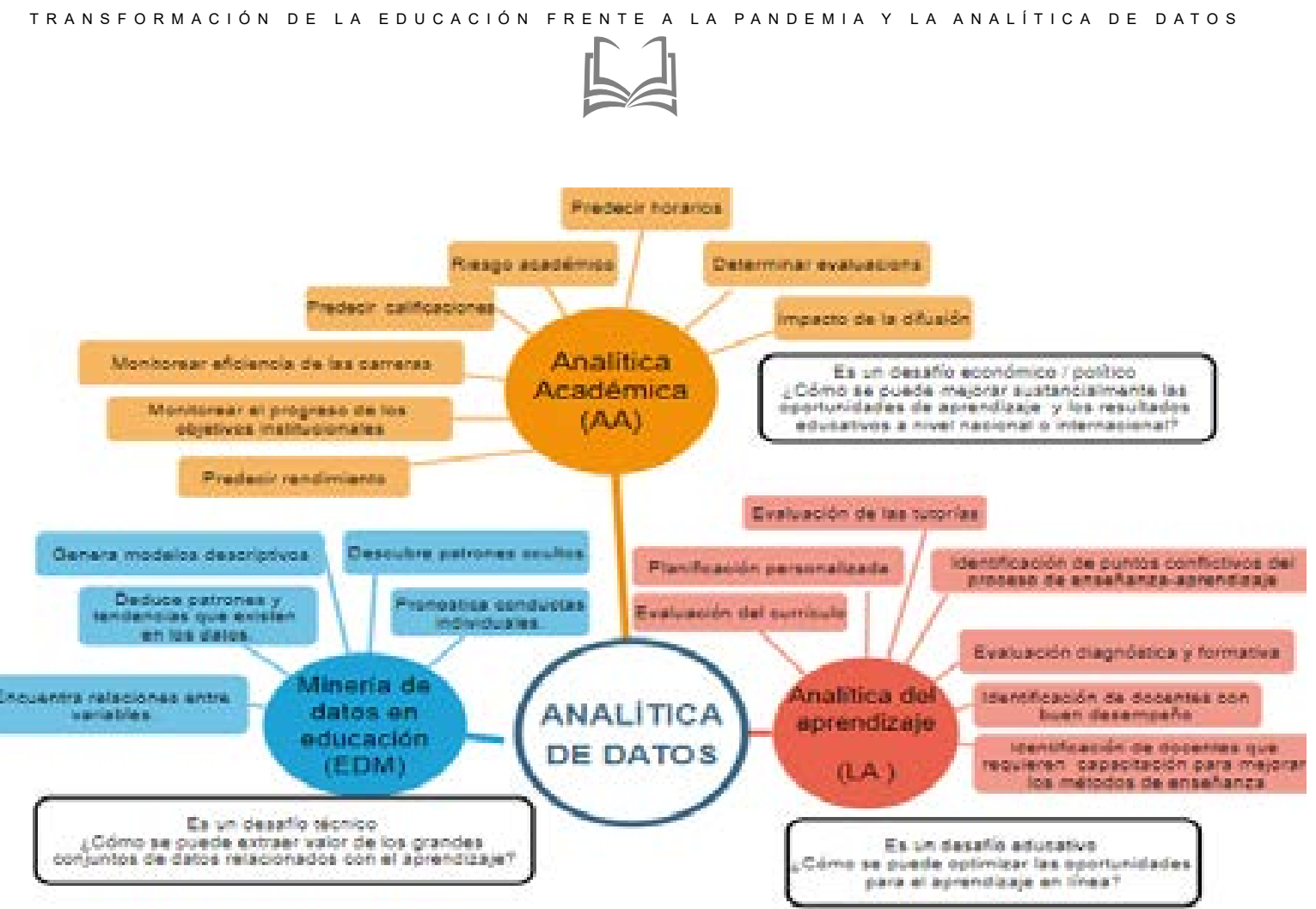

Figura 2. Enfoques de análisis de datos en educación

Ahora bien el concepto de Machine Learning aplicado a la educación puede definirse como la medición, recolección, análisis y divulgación de datos sobre los actores educativos, con el propósito de comprender y optimizar aspectos relacionados del proceso enseñanzaaprendizaje (Dyckhoff, Zielke, Bültmann, Chatti, \& Schroeder, 2012).

\section{PROPUESTA EN EL ÁMBITO DE LA EDUCACIÓN}

Autores como Khalil et al. (2015) manifiestan que la analítica del aprendizaje permite usar los datos asociados con el aprendizaje de los estudiantes y generar informes que sean útiles para los docentes (actividades y progreso de los estudiantes), para los estudiantes (retroalimentación) y para los administradores (incremento de aulas de clase, tasa de graduación, etc.) (Khalil, Khalil, \& Ebner, 2015). Los tres enfoques pueden ser utilizados en el campo de la educación a partir de los datos que se generan en plataformas y demás con la finalidad de predecir, mejorar o facilitar la toma de decisiones tanto de estudiantes, docentes, así como de las instituciones.

De los enfoques mencionados en la Figura 2, es posible encontrar diversas investigaciones, pero el estado de arte permite evidenciar escasos estudios en el enfoque "learning analytics", es decir, variables que se generen en el actuar de los estudiantes con las plataformas y recursos en línea. Esta puede ser una buena oportunidad para aportar a este campo de la investigación. Ante el panorama del uso de machine learning en el campo educativo aún hay tópicos que pueden ser fortalecidos como el uso de conjuntos de datos más consistentes y versátiles, y el uso de técnicas de minería de datos híbridas (Majeed \& Naaz, 2018). 


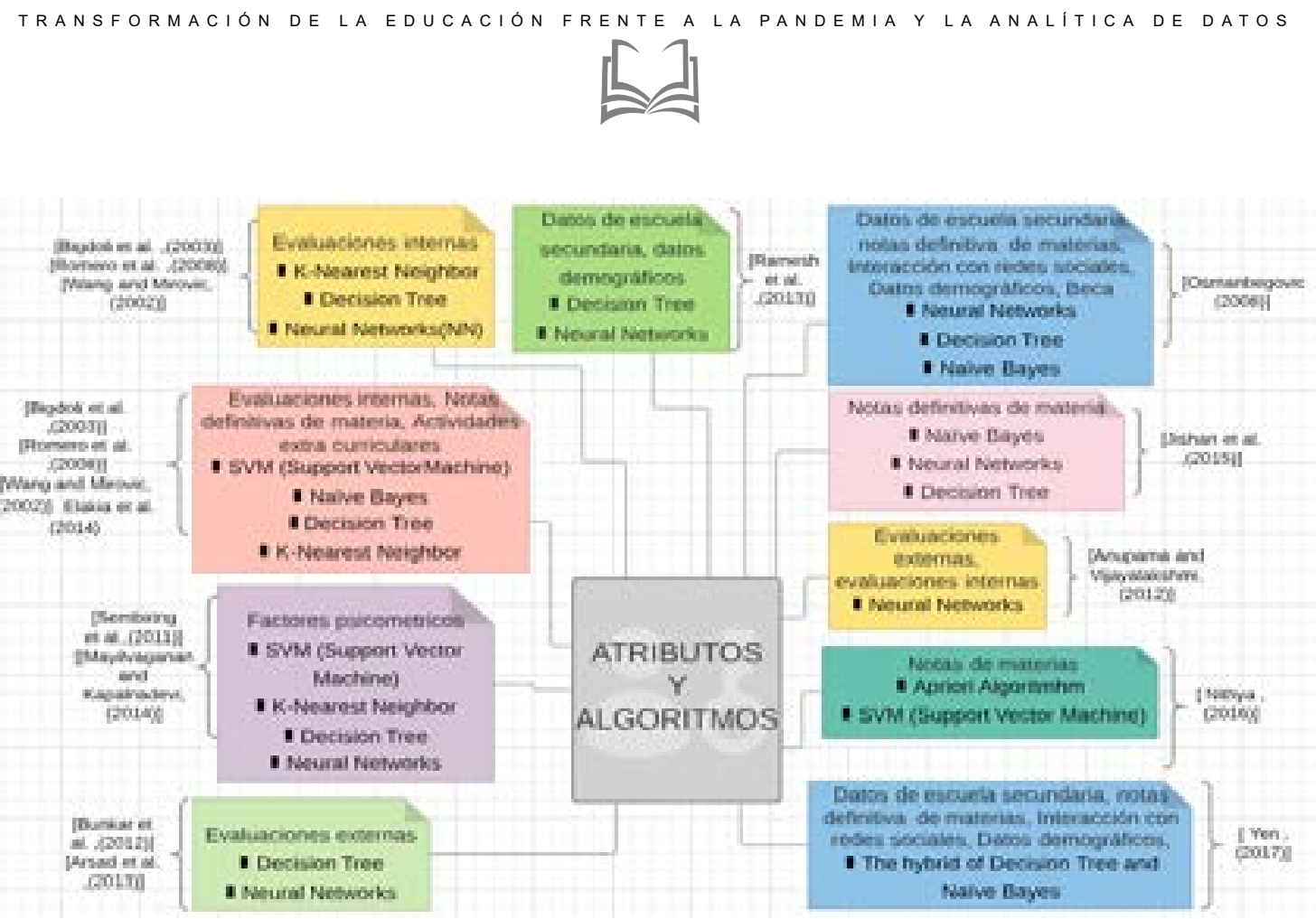

Figura 3. Algunos algoritmos usados en el campo educativo.

Fuente: Adaptado de (Shahiri et al., 2015)

Plantear la solución al problema de crear conocimiento a partir de datos, parece ser un problema de complejidad (Torres, 2017). Se propone entonces, un modelo la integración de datos provenientes del estudiante, los docentes

y la universidad con el fin de tratarlos, analizarlos y visualizarlos con el ánimo de apoyar la toma de decisiones (Figura 4).

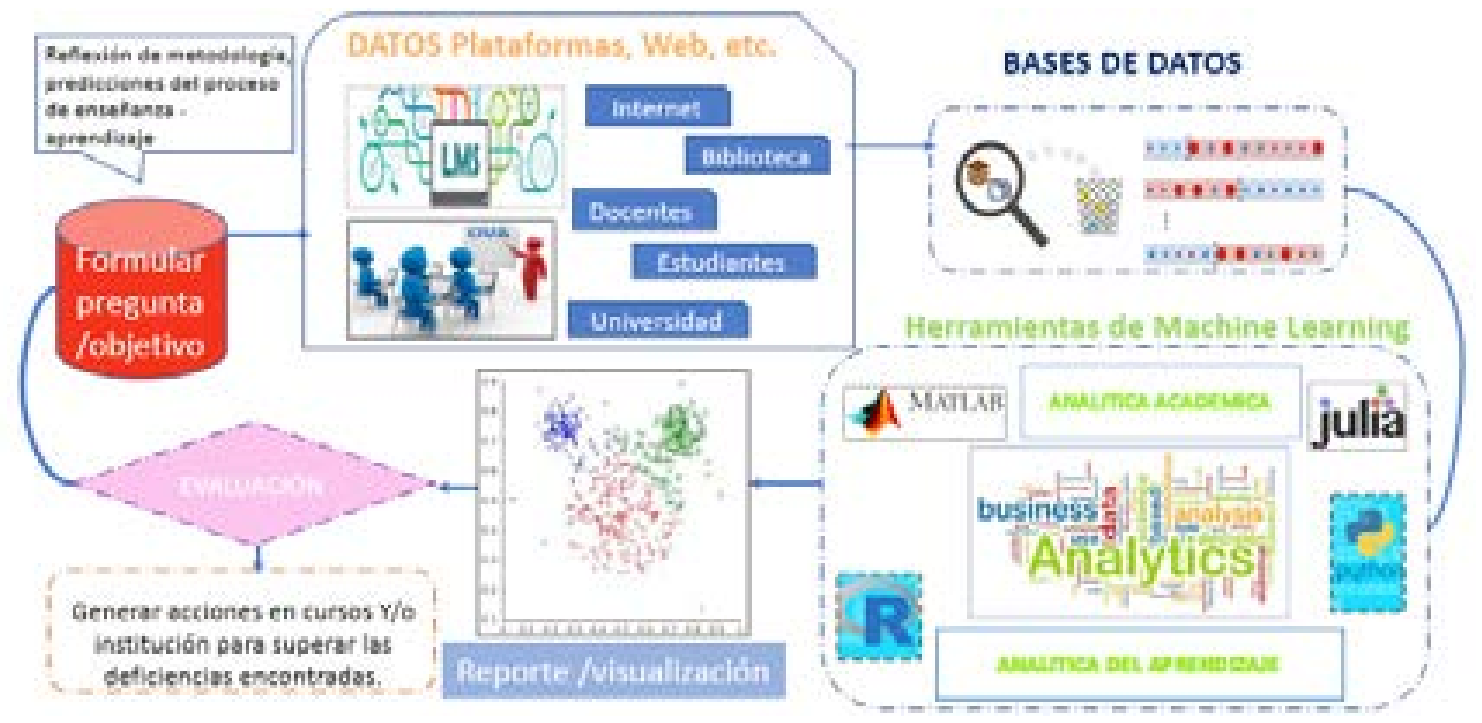

Figura 4. Modelo de usos de datos de enseñanza presencial apoyada con recursos digitales

La deserción constituye un fenómeno importante de estudio, no solo por las consecuencias económicas y sociales que representa para un país, sino por las consecuencias personales que el abandono de los estudios trae a los estudiantes y sus familias (Gardner, Dussán, \& Montoya, 2015)así como establecer las variables determinantes en el riesgo de deserción en los 
años 2012-2013. Se trabajó con una muestra no probabilística de los sujetos que respondieron al llamado o al correo electrónico enviado a todos los individuos que fueron estudiantes de la Universidad de Caldas, pero no renovaron su matrícula en algún semestre del periodo 2012-2013. Tal muestra estuvo constituida por 1005 personas, de un total de 4473 reportados por la Oficina de Planeación de la Universidad de Caldas. El $30.0 \%$ de los estudiantes encuestados argumentó el abandono de sus estudios por dificultades económicas, el $25.9 \%$ por elección equivocada de carrera que llevó a la pérdida de motivación, el $0.8 \%$ indicó problemas institucionales, el 2.1\% argumentó su deserción por motivos relacionados con el embarazo y el $0.7 \%$ abandonó los estudios por razones asociadas a una enfermedad psiquiátrica. Abstract This paper shows the findings of a descriptive research no experimental which objective was to characterize the population of students who did not renew enrollment in undergraduate programs at the University of Caldas, as well as set the variables determining the risk of desertion in the years 2012-2013. We worked with a nonrandom sample of subjects who answered the call or e-mail sent to all individuals who were students at the University of Caldas, but did not renew their enrollment in any semester period 2012-2013. This sample consisted for 1005 people out of a total of 4473 reported by the Office of Planning at the University of Caldas. $30.0 \%$ of Aproximación causal al estudio de la deserción en la Universidad de Caldas. Periodo 2012-2014* //Aproximação causal ao estudo da deserção na Universidad de Caldas. Artículo derivado de la investigación: I"La deserción estudiantil en la Universidad de Caldas: sus características, factores determinantes y el impacto de las estrategias institucionales de prevenciónl". Proyecto adscrito a los grupos de inves-tigación: Desarrollo Humano, Estadística y Matemáticas, Cognición y Educación de la Universidad de Caldas. Periodo 2012-2014.
Magister en Desarrollo Educativo y Social de la Universidad Pedagógica Nacional.

\section{CONCLUSIONES}

La coyuntura impuesta por la pandemia del COVID-19 ha cambiado para siempre lo que conocíamos como vida "normal". Ni el trabajo, ni la educación serán las que solían ser. La educación mediada por el uso de herramientas que apoyan el proceso tradicional, permitirán mediante el análisis de sus datos adelantar investigaciones de impacto. De esta manera explorar, describir e interpretar fenómenos educativos de diversa índole usando machine learning y sus algoritmos puede generar conocimiento útil para la toma de decisiones, en particular, la incidencia en la salud mental, no solo de estudiantes sino también de docentes que hoy se enfrentan a ambientes inciertos que actúan como disparadores emocionales de estrés y frustración.

El uso de plataformas en tiempos de aislamiento obligatorio permiten la comunicación de forma síncrona o asíncrona, sin limitaciones espaciotemporales entre compañeros "de clase" y docentes; el soporte de parte de los profesores se da por medio de correo electrónico, chats de texto y voz, mensajes, foros de discusión o incluso videoconferencias, de esta manera se sobrelleva la inesperada situación originada por la imposibilidad de la presencialidad.

Finalmente el modelo presentado incluye la recopilación de datos del proceso educativo generados por diferentes fuentes y actores. Esos datos se almacenan en bases de datos y a partir de herramientas de machine learning es posible extraer información que permite tomar decisiones en procura de generar acciones para superar deficiencias. El ciclo vuelve a iniciar con la formulación de una pregunta u objetivo que se pretenda responder. Para el caso particular, descubrir a partir de datos, respuesta a causas de deserción como el rendimiento 
académico, dificultades financieras, deficiencia en conocimientos previos, características individuales, falta de vocación, insuficiente tiempo dedicado al estudio, asignaturas reprobadas, desmotivación, problemas familiares, problemas de infraestructura técnica, frustración por uso de TIC, entre otros, permite tomar decisiones acertadas y oportunas.

\section{REFERENCIAS BIBLIOGRÁFICAS}

Ayesha, Shaeela; Mustafa, Tasleem; Sattar, Ahsan Raza; Khan, M. I. (2010). Data Mining Model for Higher Education System. European Journal of Scientific Research, 43, 24-32.

Contreras, L. E., Rodriguez, J., \& Tarazona, G. (2017). B-Learning and Big Data: Use in Training an Engineering Course. Lecture Notes in Computer Science, 10387, 221233. https://doi.org/10.1007/978-3-31961845-6_23

Dyckhoff, A. L., Zielke, D., Bültmann, M., Chatti, M. A., \& Schroeder, U. (2012). Design and implementation of a learning analytics toolkit for teachers. Educational Technology and Society, 15(3), 58-76.

Gil Olivera, N. A. (2019). Ambiente virtual de aprendizaje: beneficios y ventajas para enseñanza del francés como L2. Revista Boletín Redipe, 8(11), 91-99. https://doi. org/10.36260/rbr.v8i11.852

Arboleda Aparicio, J. C. (2020). Educar para la evolución de la vida humana y planetaria. Una perspectiva comprensivo edificadora. Revista Boletín Redipe, 9(6), 51-65. https://doi.org/10.36260/rbr. $\underline{\text { v9i6.998 }}$
Gardner, L., Dussán, C., \& Montoya, D. (2015). Aproximación causal al estudio de la deserción en la Universidad de Caldas. Revista Colombiana de Educación, 70, 319-340.

González, K. (2019). E-research y tecnologías colaborativas en la educación superior (Firts edit; Redipe- Red Iberoamericana de pedagogía, Ed.). USA.

Keswani, R. N., Sethi, A., Repici, A., \& Chiu, P. (2020). How To Maximize Trainee Education During the COVID-19 Pandemic: Perspectives from Around the World. Gastroenterology. https://doi.org/https:// doi.org/10.1053/j.gastro.2020.05.012

Khalil, M., Khalil, M., \& Ebner, M. (2015). Learning Analytics: Principles and Constraints. EdMedia: World Conference on Educational Media and Technology, 2015(1), 1789-1799.

Litzinger, T. A., Lattuca, L. R., Hadgraft, R. G., \& Newstetter, W. C. (2019). Engineering Education and the Development of Expertise. Journal of Engineering Education, 100(1), 123-150.

Lizárraga Juárez, A., López Ramírez, E., \& Martínez Iñiguez, J. E. (2020). Apropiación tecnológica en el manejo de competencias digitales por los profesores de escuelas normales en México. Revista Boletín Redipe, 9(6), 157-167. https://doi.org/10.36260/rbr. v9i6.1009

Majeed, I., \& Naaz, S. (2018). Current State of Art of Academic Data Mining and Future Vision. Indian Journal of Computer Science and Engineering (IJCSE), 9(April), 49-56. https://doi.org/10.21817/indjcse/2018/ v9i2/180902026 
Sanabria, A. (2011). Percepción de los estudiantes y profesores sobre el uso de las tic en los procesos de cambio e innovación en la enseñanza superior. Revista de Psicologia, Ciències de l'Educació $i$ de l'Esport, 29, 273-290.

Schroeder, U. (2009). Web-Based Learning Yes We Can! https://doi.org/10.1007/9783-642-03426-8_3

Shahiri, A. M., Husain, W., \& Rashid, N. A. (2015). A Review on Predicting Student's Performance Using Data Mining Techniques. Procedia Computer Science, 72, 414-422. https://doi.org/10.1016/J. PROCS.2015.12.157

Torres, J. (2017). Inteligencia Integral de negocios (primera; Doctorado en ingeniería Universidad Distrital, Ed.). Bogotá.

UNESCO. (2020, April). Perturbación y respuesta de la educación de cara al COVID-19. 